\title{
On liquid crystal thermography for determination of the heat transfer coefficient in rectangular ducts
}

\author{
Z. Ghorbani-Tari ${ }^{1}$, B. Sunden ${ }^{1} \&$ G. Tanda ${ }^{2}$ \\ ${ }^{1}$ Department of Energy Sciences, Division of Heat Transfer, \\ Lund University, Sweden \\ ${ }^{2}$ Dipartimento di Macchine, Sistemi Energetici e Trasporti (DIMSET), \\ Università degli Studi di Genova, Italy
}

\begin{abstract}
Liquid crystal thermography (LCT) has been employed by researchers in heat transfer and fluid flow communities as a reliable and non-intrusive temperature measurement technique. This technique correlates the colour response to temperature for a heated surface which has been treated with thermochromic liquid crystals (TLCs). The LCT has been used extensively in convective heat transfer research in duct flows. In this paper, some experiences by LCT in thermal measurements for rectangular duct flows are provided. A few TLCs examples associated with continuous ribs for two different values of rib pitch-toheight ratio of 4 and 8 at $R e=8900$ and 28500 are illustrated. Important issues such as heating principles, calibration of TLCs, image acquiring and analysis, problems of treating the surface by TLCs, and expected measurement accuracy are summarized. The main purpose is to assist newcomers in the field and provide some guidelines for proper use of the LCT in heat transfer research.
\end{abstract}

Keywords: LCT, convective heat transfer, measurement accuracy.

\section{Introduction}

Liquid crystal thermography (LCT) has emerged as reliable measurement sensors for the visualization and determination of surface temperature distributions leading to convective heat transfer coefficients [1]. The advantages of LCT are, e.g., its flexibility to be used from micron sized electronic circuits to 
large scale gas turbine blades, high spatial resolution, steady state and transient temperature measurements, temperature accuracy $\pm 0.1{ }^{\circ} \mathrm{C}$, optical system based on visible light and independent of surface emissivity, ability to use with common color video and CCD cameras and conventional recorders, cost effective compared to infrared thermography (IR) systems etc. The LCT has been used extensively in convective heat transfer research in duct flows (applications in heat exchangers, and internal cooling of gas turbines and electronics cooling). References [2-4] are examples of published papers. Descriptions of LCT measurement technique can be found in, e.g., refs. [5-8].

The LCT correlates the color response to temperature for a heated surface which has been treated with thermochromic liquid crystals (TLCs). The TLCs are complex organic substances that once illuminated with a white light; reflect visible light of a narrow frequency band depending on temperature. The dominant reflected wavelength decreases with increasing temperature of the TLCs: red color appears at the lowest temperature of the range of indicated temperatures and blue at the highest temperature [9]. This behavior is exhibited within a range of temperatures called event temperature range. The TLCs with an event temperature range within $5-30^{\circ} \mathrm{C}$ are termed broad band and those within $0.5-4{ }^{\circ} \mathrm{C}$ are termed narrow band [5]. Bandwidth as small as $0.1^{\circ} \mathrm{C}$ has also been reported [10]. The use of narrow band TLCs is an attractive alternative for temperature and heat transfer measurements due to their higher accuracy in temperature measurements and also the fact that narrow band TLCs are less affected by variation in illumination-viewing angles and illumination disturbances [5]. For full field measurements where the surface temperature is non-uniform, the narrow band TLC cannot be used directly but instead a more tedious procedure is needed. The wide band TLCs has better ability to record the entire surface temperature distribution from a single image, if the TLC bandwidth is large enough to span all temperatures experienced on a test surface. However, the primary drawback is that wide-band TLCs require a significant number of calibration points to accurately resolve the highly non-linear variation of hue versus temperature of typical TLC [11].

In this paper, calibration of TLCs, image acquiring and analysis, some important issues like heating principles (e.g., how to ensure uniform heating), and problems of treating the surface by TLCs are summarized. Some examples of TLCs images for a straight rectangular duct equipped with arrays of continuous ribs at different Re values are illustrated. Two different values of rib pitch-to-height ratio of 4 and 8 are considered to demonstrate heat transfer coefficients distribution on the end wall. The main purpose is to assist newcomers in the field and provide some guidelines for proper use of LCT in heat transfer research.

\section{Thermochromic liquid crystals - literature review}

The TLC compounds are difficult to deal with under most heat transfer laboratory conditions. Several adverse effects are present in any laboratory or field setting such as chemical contamination and exposure to ultra-violet (UV) 
light which primarily degrade the colour response. Several approaches have been developed to make TLCs more practical to use [12]. Micro-encapsulation is the most popular method of protection and the liquid crystal is enclosed in polymer spheres which can be as small as a few microns. Commercial micro-encapsulated TLCs may be purchased in water-based slurry and are generally applied to flat or curved surfaces using an air-brush spray. The thickness of coating must be controlled carefully and further reading can be found in ref. [13, 14]. The most convenient manner to measure the thermal field on a surface is to coat it with a pre-packaged assembly consisting of a liquid crystal layer painted on a plastic sheet having a background colour with black ink [12]. The pre-packaged assembly TLC is limited in application because they are not typically available in a sprayable medium and limited to relatively flat surfaces. Edge effects due to chemical contamination may destroy the pre-packaged surface when a cutout portion of a manufactured sheet is used. To be used in advanced implementations like research on heat transfer coefficients on duct walls one needs to heat the surface of interest, apply calibration for the color-temperature response, acquire images and analyze the images by suitable software for image processing. Huebased image processing [15] is the most common technique used in applications of LCT to interpret the TLC signal. The color of TLCs observed by a RGB-data (red-green-blue) acquisition system is transformed to hue (H), saturation (S) and intensity (I) color space. HSI color space provides approximately the human description of color [1]. Hue is an optical attribute related to actual colour of the point of observation [12]. Hue is the only among these parameters being retained due to its monotonic relationship with surface temperature [16]. The hue value of each pixel indicates the temperature of corresponding location on the surface. This property of hue and its independence of the light intensity levels make it suitable for temperature measurements [17].

In experimental heat transfer, one demanding issue related to liquid crystals is the colour-temperature relationship, and any quantitative application of TLCs requires an accurate calibration [13]. It is essential to keep the lighting and viewing conditions of the thermal experiments identical to the calibration experiment as a colour pattern can be significantly affected by lighting and viewing angles [12]. There are several papers related to TLC calibration and the main factors being pertinent to calibration. Some of these are summarized here. The illumination source has a significant effect on the shape of the huetemperature calibration curve and this may lead to higher or lower temperature uncertainties over the applicable range. If the hue-temperature curve is a straight line, this would be the sensitivity at every hue; however, hue-temperature curves have regions of higher and lower sensitivity [18]. The background lighting is another important factor which may influence the shape of the hue-temperature calibration curve [18]. The hysteresis is characterized by a decrease in reflectivity and a shift in temperature associated with the peak reflected intensity for each of R, G and B components during cooling. This causes a shift in the hue-temperature relation of the TLC. This results in temperature biases when cooling occurs instead of heating [19]. The hysteresis is not a limiting measurement error factor if the temperature of a measured surface remains 
within or below the active range of the TLC [17]. Conversely, the magnitude of the hysteresis can be significant if the temperature exceeds the TLC active range even for short periods of time [17, 19]. The magnitude of hysteresis and aging effects is related to the bandwidth; smaller the bandwidth greater the effect [19]. Ref. [17] reported that a thickness of the TLC layer affected the calibration and vulnerability to aging, i.e., a change in the hue-temperature relationship with time due to prolonged exposure to high temperature and usage. Ref. [19] reported a similar effect; the permanent damage to TLCs during exposure to temperatures above the clearing point. The sensitivity to aging and damage is shown to be more acute for thinner film thicknesses [13]. The reading of the TLC colours depends on the angles between the light source and the TLC surface, and between the camera and the TLC surface [17]. Further details about the effects of lighting and viewing arrangements on TLCs colour can be found in, e.g., refs. $[15,17,20]$. The perceived color of a TLC depends on the optical properties of the measurement path and the reader is referred to refs. $[13,20]$.

\subsection{Selection of calibration methods}

The calibration experiment can be conducted in a separate system (on which controlled thermal conditions are imposed) or directly in a test surface (in-situ), using the same optical arrangement as thermal measurement section [12]. The calibration conducted in a separate system may result in some problems. This might be due to the situation imposed in the actual thermal measurements and it might be difficult to enable the similar conditions precisely for the calibration. The in situ calibration might be more beneficial as it is referred by refs. $[11,21]$. However, the arrangement of in situ calibration is more complicated because several thermocouples have to be fitted into a test section. Therefore, it can be

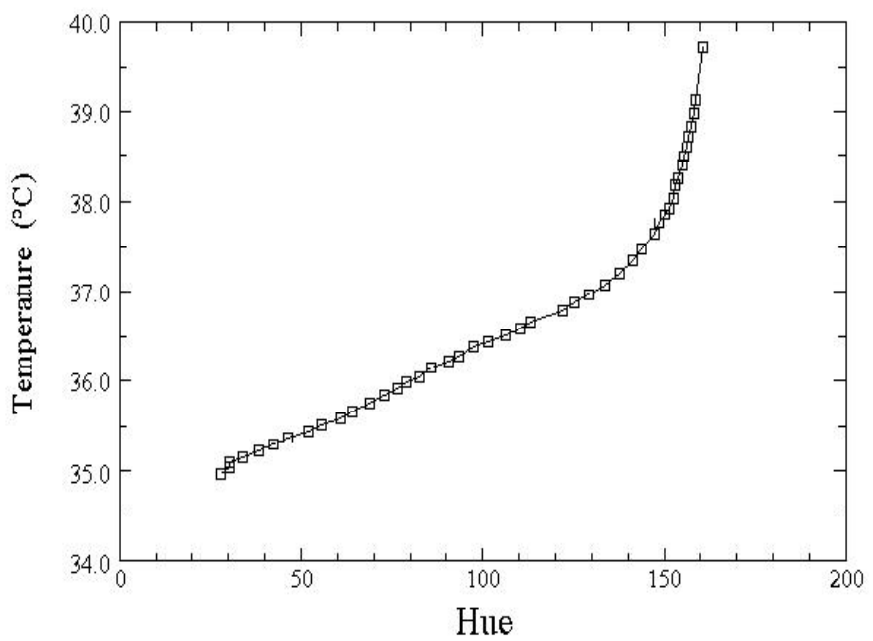

Figure 1: $\quad$ Hue-temperature calibration [22]. 
more practical to make calibration on a separate plate where several thermocouples are fitted and the thermally boundary conditions can be more easily controlled.

Fig. 1 shows an example of a typical hue-temperature calibration curve. The process of calibration is omitted here and the reader is referred to ref. [22]. The active range starts at a temperature of $35^{\circ} \mathrm{C}$ with a bandwidth of $5{ }^{\circ} \mathrm{C}$. The hue is fairly linear and repeatable in the range of 60-120 and thus can be adopted for the reconstruction of heat transfer coefficient distribution. The linear portion of the hue field is usually adopted due to its higher sensitivity; sensitivity at every hue value, and thus is reliable to be applied in thermal measurements.

\section{Selection of heat measurement method}

Two commonly used methods for heat transfer measurement are steady state where experimental properties do not vary with time and transient liquid crystal method in which heat transfer coefficients are deduced from a time-temperature history record [1]. The steady state heat transfer experiment with a constant heat flux density is conceptually simple and relatively easy to analyze. The heat transfer coefficient is obtained from dividing the heat flux by the measured temperature difference between the TLC surface and the fluid. However, the steady state method requires the maintenance of a uniform surface heat flux and this attainment might be difficult even by using an electric heating foil. The maximum heat flux must not cause an appreciable temperature difference across the TLC layer either. For the transient method a surface temperature is subject to a step change in the fluid flow temperature. The surface is then considered to be the interface between the fluid and a semi-infinite body, which implies that an analytical solution for the transient heat conduction problem can be used to evaluate the heat transfer coefficient. If a surface cannot be considered as the boundary of a semi-infinite body but must be regarded as a thin layer, then the temperature is assumed to be uniform in the lateral direction. A lumped heat transfer analysis is then performed in the evaluation of the convective heat transfer coefficient.

\subsection{Steady state-constant heat flux}

The steady state thermal measurement is employed and TLCs are used to monitor the surface temperature. The test section coated by heating foil is electrically heated to maintain the surface temperature greater than the air temperature, i.e., $\mathrm{T}_{\mathrm{LC}}>\mathrm{T}_{\mathrm{a}}$, and convective heat transfer occurs between the surface and the fluid. The surface electric heater is employed such that local heat flux is known and this, together with the local surface temperature gives the local heat transfer coefficient [23]. The important issue is that the applied heating foil provides a uniform heat flux through the test section. If TLC is attached to the heating foil directly, the heat flux might be more concentrated over the electrical path and that may lead to a non-uniform heat flux distribution over the test surface, see fig. 2 (a). One way to solve this might be to apply a thin metallic 
plate to which a plane heater is attached and then a more uniform heat flux is obtained, see fig 2 (b). The thermal conductivity of the selected metallic plate may not be too high because then a uniform wall temperature distribution might be achieved instead. The thermal contact resistance is another important issue. This occurs when different conducting surfaces, e.g., heating foil, metallic foil, TLC are not fit tightly together and a thin layer of air is trapped between them. As a consequence, heat is transferred by conduction through the contact points of the solid, while convection and radiation take place across the trapped interfacial air and result in a temperature drop across the interface and this cannot be ignored. The contact resistance is a function of pressure holding different surfaces and this highlights the importance of which metallic interface should be considered to increase the pressure and thus reduce the thermal contract resistance. As a result, one should always be aware of the existence of the interface resistance and the resulting temperature difference across the interface [24].

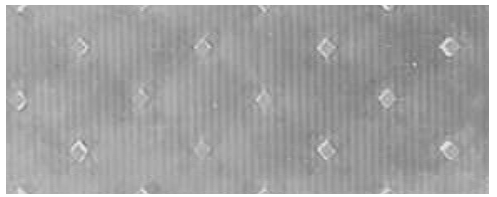

(a)

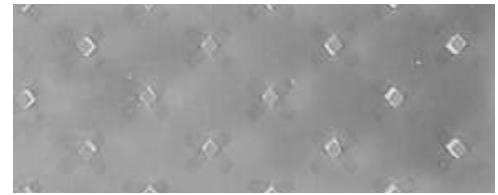

(b)

Figure 2: TLC directly attached to a plane heater: the electric tracks are clearly visible, symptom of a non-uniform heat flux at surface (a); A thin stainless steel between TLC and the plane heater: the heat flux is more evenly distributed over the surface (b).

\section{Thermal measurement}

\subsection{Thermal test section apparatus}

The experimental apparatus is not shown here for the sake of brevity and the reader is referred to ref. [16]. The test section is a rectangular duct as wide and high as the entry and exit sections and delimited by a thin heated plate $(0.1 \mathrm{~m}$ wide and $0.28 \mathrm{~m}$ long) lateral and frontal Plexiglas walls. The heated side of the test section consists of $0.5 \mathrm{~mm}$-thick stainless steel foil to which a plane heating foil is glued. A thin TLC sheet is applied on the stainless steel foil, on the side exposed to the air flow, to measure local surface temperature. The rear side of the heated plate is thermally insulated to convey as much as possible of the electric power dissipated by the heater to the convective air flow. Electric power is supplied by a DC to measure voltage and current of the heater. Fine-gauge thermocouples were placed inside the rectangular duct, directly exposed to the airflow, and at several sites inside the duct wall material. These sensors are used to measure the air temperature at the test section inlet, to estimate conduction heat losses to the surroundings and to control the attainment of the steady state 
conditions. The TLC used here is supplied by pre-packaged sheet $(0.15 \mathrm{~mm}$ thick) to measure temperature distribution on the heated surface. The colour distribution of TLC is observed by a CCD video camera through the Plexiglas wall opposite to the heated surface and stored in a PC. The light for the illumination is switched on only for the time required for image acquisition to avoid radiant heating of the test surface [16].

\subsection{Measuring procedure}

The test surface is roughened by insertion of multiple ribs. The airflow is adjusted to a prescribed velocity and at the same time DC current is supplied to the heater. The test surface temperature is maintained within the linear portion of the calibration curve by controlling the input power delivered to the heater and therefore no information is gained outside that region. That necessitates repeating the tests at the same flow rate and for several different values of the input power to move the color pattern toward the regions not previously monitored and to extract local the heat transfer coefficients $(h)$, eqn (1). One important issue is that the input power and TLC images are recorded at steady state. The heat transfer coefficient distributions from each test (at a given heat flux) are superimposed on some regions of the test surface and an average value is taken with the requirement that the discrepancy between $\mathrm{h}$ values for the same pixel never exceeds $2 \%$ [16].

$$
h=\frac{Q_{e l}-Q_{l o s s}}{A \cdot\left(T_{L C}-T_{b}\right)}
$$

The convective heat flux is assumed to be uniformly distributed and it is obtained by measuring input power to the heater $\left(Q_{e l}\right)$ corrected by heat loss rate, divided by the difference between surface temperature detected by the TLC and air bulk temperature $\left(T_{b}\right)$ at the current position along the streamwise direction. The surface area $(A)$ is assumed as that of the smooth surface. The heat loss rate is calculated by radiation, conduction (through the insulation on the rear face of the heater) and heat dissipation from the ribs. Although, the ribs made of Plexiglas are deemed adiabatic due to low thermal conductivity, the conventional one-dimensional fin model is applied to estimate the small amount of heat transfer rate from the rib sides to the air flow [16].

\subsection{Heat transfer coefficients for the ribbed duct}

Continuous ribs are positioned on the surface of the duct (end wall) at periodic sites and oriented transversely to the main flow direction. The geometric parameters (heated surface length $\mathrm{L}$ and width $\mathrm{W}$, channel height $\mathrm{H}$, rib height $\mathrm{e}$, rib pitch $\mathrm{p}$, streamwise rib length 1 ) are summarized in table 1 . The rib pitch 20 and $40 \mathrm{~mm}$ are considered for the demonstrated configurations. The height and the streamwise length of the ribs were kept fixed at 5 and $3 \mathrm{~mm}$, respectively. 
Table 1: Geometric parameters of rib configurations [16].

\begin{tabular}{|c|c|c|c|c|c|c|c|}
\hline config. & L (mm) & W (mm) & H (mm) & l (mm) & p (mm) & e (mm) & p/e \\
\hline A & 280 & 100 & 20 & 3 & 20 & 5 & 4 \\
\hline B & 280 & 100 & 20 & 3 & 40 & 5 & 8 \\
\hline
\end{tabular}

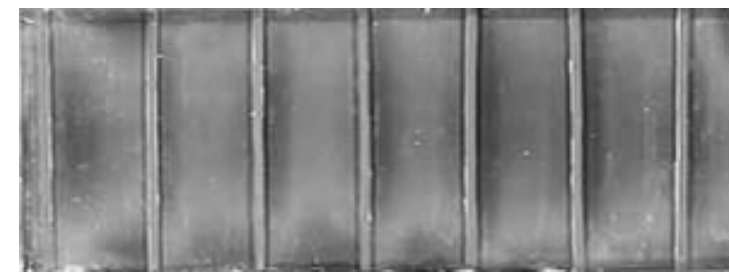

Figure 3: $\quad$ TLC image for configuration B [16].

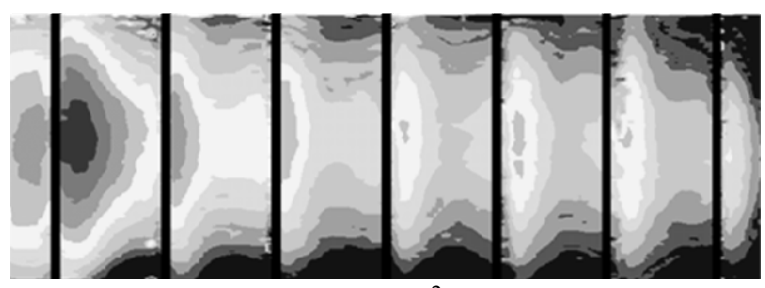

$\mathrm{H}\left[\mathrm{W} / \mathrm{m}^{2} \mathrm{~K}\right]$

\section{$\begin{array}{llllllllll}\| 40.42 & \| 42.44 & \| 44.46 & \| 46.48 & 48.50 & \| 50.52 & \| 52.54 & \| 54.56 & \| 56.58 & \| 58.00\end{array}$}

Figure 4: 2-D plot heat transfer coefficient $\left(\mathrm{W} / \mathrm{m}^{2} \mathrm{~K}\right)$ for configuration at $\mathrm{Re}$ $=8900[16]$.

An example of a TLC image taken by a CCD video camera for configuration $\mathrm{B}$ at the Reynolds number $(\operatorname{Re}=8900)$ and a specific heat flux is illustrated in fig. 3. As explained previously, only points within the linear portion of the calibration curve is taken from some images like the one illustrated in fig. 3 and processed for the reconstruction of full field heat transfer coefficients, as shown in fig. 4. The inter-rib h-distribution at midline is demonstrated in fig. 5 for configurations A and B, respectively [16].

Inspection of fig. 5 shows that for the configuration $A(p / e=4)$, the inter-rib h-distribution is characterised by a monotonic increase that periodically repeats itself after the third-fourth rib. This probably indicates the presence of a trapped vortex between ribs without a reattachment point. Conversely, for configuration 
Configuration A

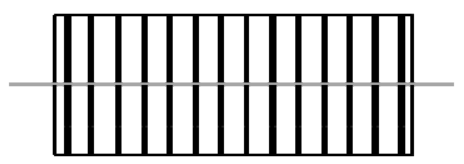

Configuration B

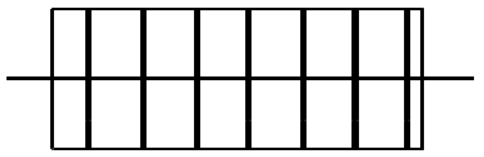

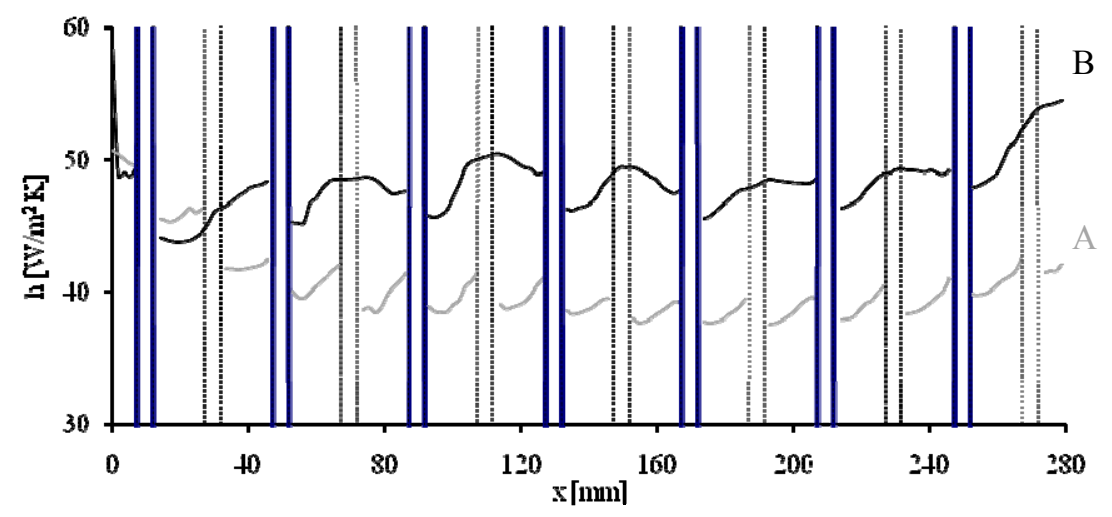

Figure 5: Distribution of local center-line heat transfer coefficient for the continuous rib configuration $\mathrm{A}$ and $\mathrm{B}$ at $\mathrm{Re}=28500$ [16].

$\mathrm{B}(\mathrm{p} / \mathrm{e}=8)$, the heat transfer coefficient reaches a maximum value at the point of flow reattachment. Downstream of the reattachment point, the heat transfer coefficient slightly decreases up to the vicinity of the successive rib. The periodic fully developed conditions are attained approximately after the third rib [16].

\section{Estimation of uncertainties}

Several factors (image noise, lighting angle, TLC coating thickness and coating quality) may influence the hue-temperature relationship and the expected measurement accuracy. In addition, the uniformity of the heating procedure and various sources of losses to the surroundings may influence the accuracy of the measurement. The image noise can be reduced by using filtering techniques to erase hue spots not related to temperature and that leads to improvement in accuracy of the measurements. The lighting angle is known to have a significant effect on TLCs hue-temperature curve and thus on the accuracy of the measurements. Therefore, it is important that the lighting set up in a real measurement is the same as at the calibration stage. The coating thickness has a significant effect on a hue-temperature curve but it has been found to have a nondistinctive effect on the measurement uncertainty. The coating quality affects both the hue-temperature curve and the accuracy of the measurement. Further reading is available in ref. [6]. In applications where the overall purpose is to determine convective heat transfer coefficients, other uncertainties impact on the 
final accuracy; i.e., the uncertainties in flow rate or Reynolds number and the flow temperatures. Consideration of all uncertainties is commonly taken into account applying the root-sum-square combination as described in ref. [25].

\section{Concluding remarks}

In this paper, some important issues related to the use of TLCs for heat transfer measurements in duct flows (treatment of the surface, TLC calibration, image acquiring and analysis, etc.) are summarized. Regardless of calibration method, each TLC has its own calibration curve. The linear portion of the calibration curve is recommended to be adopted due to higher sensitivity and thus more reliable for thermal measurements. Concerning the thermal boundary condition typically adopted in the LCT, steady-state, heat measurement method, and care should be taken in the use of an electrically heating foil to attain a uniform heat flux; often the insertion of a thin metallic plate between the heater and the TLC sheet could improve the uniform distribution of heat flux at the wall. Another concern associated with the heating issues is the thermal contact resistance and resulting temperature drop when different conducting surfaces, e.g., heating foil, metallic foil, TLC are not in perfect thermal contact.

There are several factors which impact the accuracy of thermal measurements associated with the LCT technique and some of those were outlined in this paper. The main purpose was to highlight some important issues collected from several researchers to assist newcomers and provide some guidelines for proper use of $\mathrm{LCT}$ in heat transfer research.

\section{Acknowledgements}

Part of this work is financially supported by the Swedish Energy Agency, Volvo Aero Corporation and Siemens Industrial Turbines through the national Swedish research program TURBO POWER, project TURB3. The work is also supported by a separate project by the Swedish Energy Agency.

\section{Nomenclature}

$\begin{array}{ll}A & \text { heat transfer area } \\ \mathrm{e} & \text { rib height } \\ h & \text { heat transfer coefficient } \\ \mathrm{H} & \text { channel height } \\ \mathrm{HSI} & \text { hue, saturation, intensity } \\ \mathrm{L}, \mathrm{W} & \text { length and width of the heated section } \\ \mathrm{l} & \text { length of the rib } \\ \mathrm{p} & \text { rib pitch } \\ Q_{e l} & \text { input power to the heater } \\ Q_{\text {loss }} & \text { heat loss rate by radiation and conduction } \\ \mathrm{Re} & \text { Reynolds number }\end{array}$


RGB red, green, blue

$T_{b} \quad$ air bulk temperature

$T_{L C} \quad$ surface temperature detected by TLCs

\section{References}

[1] Chan, T.L., Ashforth-Frost, S. \& Jambunathan, K. , Calibrating for viewing angle effect during heat transfer measurements on a curved surface, Int. J. Heat and Mass Transfer , 44, pp. 2209-2223, 2001.

[2] Sunden. B., On heat transfer and fluid flow in ribbed ducts using liquid crystal thermography and PIV measurements, EXHTF-7(CD-ROM Proceedings), 139-152, 2009.

[3] Tanda. G., Heat transfer in rectangular channels with transverse and Vshaped broken ribs, Int. J. Heat Mass Transfer, 47, 229-243, 2004.

[4] Wang, L. \& Sunden, B., Experimental investigation of local heat transfer in a square duct with continuous and truncated ribs, Experimental Heat Transfer, 18, 179-197, 2005.

[5] Abdullah, N., Talib, A.R.A., Jaafar, A.A., Salleh, M.A.M. \& Chong, W.T, The basics and issues of thermochromatic liquid crystal calibrations, Exp. Thermal Fluid Science, 34, 1089-1121, 2010.

[6] Rao, Y. \& Zang, S., Calibrations and measurement uncertainty of wideband liquid crystal thermography, Meas. Sci. Technol., 21, paper no. $015105,2010$.

[7] Ireland, P.T. \& Jones, T.V, Liquid crystal measurements of heat transfer and surface shear stress, Meas. Sci. Technol., 11, 969-986, 2000.

[8] Critoph, R.E., Holland, M.K. \& Fisher, M., Comparison of steady state and transient methods for measurement of local heat transfer in plate fin-tube heat exchangers using liquid crystal thermography with radiant heating, Int. J. Heat Mass Transfer, 42, 1-12, 1999.

[9] Behle, M., Schulz, K., Leiner, W. \& Fiebig, M., Colour-based image processing to measure local temperature distributions by wide-band liquid crystal thermography, Applied Scientific Research, 56, pp. 113-143, 1996.

[10] Grodzka, P.G. \& Facemire, B.R. Tracking transient temperatures with liquid crystals, Letters Heat Mass Transfer, 2, pp. 169-178, 1975.

[11] Smith, C.R., Santino, D.R. \& Praisner, T., Temperature sensing with thermochromic liquid crystals, Exp. Fluids, 30, 190-201, 2001.

[12] Tanda, G., Application of optical methods to the study of convective heat transfers in rib-roughened channels, $\mathrm{PhD}$ thesis, The City University London, 1996.

[13] Kakade, V.U, Lock, G.D, Wilson, M., Owen, J.M. \& Mayhew, J.E, Accurate heat transfer measurements using thermochromic liquid crystal. Part 1: Calibration and characteristic of crystals. Int. J. Heat Fluid Flow, 30, No. 5, pp. 939-949, 2009.

[14] Baughn, J. W., Liquid crystal methods for studying turbulent heat transfer, Int. J. Heat and Fluid Flow, 16, 365-375, 1995. 
[15] Camci, C., Kim, K. \& Hippensteele, S.A., A new hue capturing technique for the quantitative interpretation of liquid crystal images used in convective heat transfer studies, ASME J. Turbomachinery, 114, pp. 765775, 1992.

[16] Cavallero, D. \& Tanda, G., An experimental investigation of forced convection heat transfer in channels with rib turbulators by using the liquid crystal thermography, Exp. Thermal Fluid Sci., 26, pp. 115-121, 2002.

[17] Wiberg, R. \& Lior, N., Error in Thermochromic Liquid Crystal Thermometry, Review of Scientific Instruments, 75, No. 9, pp. 2985-2994, 2004.

[18] Anderson, M.R. \& Baughn, J.W., Liquid-crystal thermography: illumination spectral effects. Part1- experiments, ASME J. Heat Transfer, 127, pp. 581-587, 2005.

[19] Anderson, M.R. \& Baughn, J.W., Hysteresis in liquid crystal thermography, ASME J. Heat Transfer, 126, 339-346, 2004.

[20] Farina, D.J., Hacker, J.M., Moffat , R.J. \& Eaton, J.K., Illuminant invariant calibration of thermochromic liquid crystals, Exp. Therm. Fluid Sci. 9 (1), pp. 1-12, 1994.

[21] Baughn, J.M., Anderson, M.R., Mayhew, J.E. \& Wolf, J.D., Hysteresis of thermochromic liquid crystal temperature measurement based on hue, ASME J. Heat Transfer, 121, pp. 1067-1072, 1999.

[22] Gao, X., Heat transfer and fluid flow investigations in ribbed ducts and impinging jets using liquid crystal thermography and $\mathrm{PIV}, \mathrm{PhD}$ thesis, Division of Heat Transfer, Lund University, 2002.

[23] Incropera, F.P. \& DeWitt, D.P., Fundamentals of Heat and Mass Transfer (Chapter 7), External Flow, John Wiley \& Sons, Inc. pp. 347, 1996.

[24] Kreith, F. \& Bohn, M.S. Principles of Heat Transfer (Chapter 1). Basic Modes of Heat Transfer, Harper \& Row, New York. pp. 12-13, 1986.

[25] Moffat, R., Describing the uncertainties in experimental results, Exp. Thermal Fluid Science, 1, 3-17, 1988. 